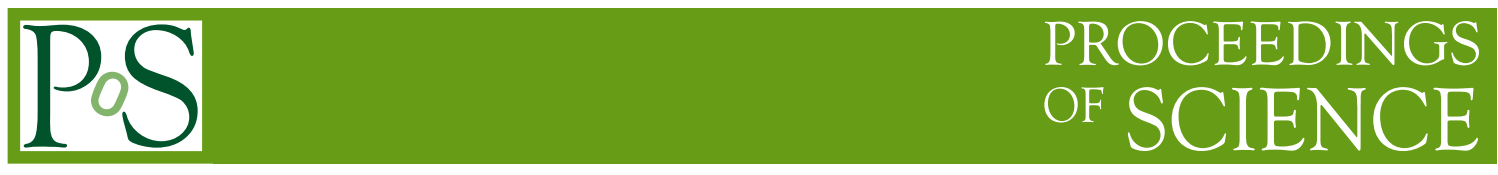

\title{
Characterizing the long-term $\gamma$-ray variability of the BL Lac object 1ES 1215+303 with Fermi-LAT
}

\section{Floriana Zefi*}

Laboratoire Leprince-Ringuet, École Polytechnique, CNRS/IN2P3, 91128 Palaiseau, France E-mail: zefiellr.in2p3.fr

\begin{abstract}
We report on the recent flaring activities from the BL Lac source 1ES 1215+303 detected by the Fermi-Large Area Telescope (LAT; $100 \mathrm{MeV}<\mathrm{E}<500 \mathrm{GeV}$ ). Since its first detection at energies above $100 \mathrm{MeV}$, several increments on the $\gamma$-ray flux have been reported, where the latest ones from 2015 to 2017 are presented in this study. Additionally, we use all the data set accumulated by Fermi-LAT spanning more than nine years, along with the improved analysis techniques to perform spectral studies and characterize the long term variability. The results obtained are presented in this contribution.
\end{abstract}

35th International Cosmic Ray Conference

10-20 July, 2017

Bexco, Busan, Korea 


\section{Introduction}

The object 1ES 1215+303, also known as ON 325 and B2 1515+30, is a BL Lac Blazar type located at a redshift of $\mathrm{z}=0.13$ [1]. It was listed as a $\gamma$-ray emitter in the first Fermi-LAT bright catalog in 2009 and was first detected at TeV energies by the MAGIC experiment in 2011 after triggering an optical high flux state [2] [3]. Since its first detection at very-high-energies (VHE), other high-flux states from this object have been reported in this energy range. Observations from Fermi-LAT covering the flares reported at VHE by VERITAS and MAGIC experiments resulted in only one simultaneous detection of a bright flare seen by VERITAS in 2014 February 14 [4]. A Doppler factor of $\delta<10$ is required to explain the extreme flare detected at VHE in 2014.

Blazars are Active Galactic Nuclei, whose relativistic jets are aligned closely to the observer's line-of-sight. They are known to undergo extreme and high-amplitude variable emission across the electromagnetic spectrum [5]. The spectral energy distribution (SED) is characterized by a doublehump structure with one peak located in the radio-to-UV/X-ray range and a second one in the X-to$\gamma$-ray range. The first peak is attributed to the synchrotron radiation from the relativistic electrons moving in the jet's magnetic field. The origin of the second component is still under debate where two different scenarios of hadronic or leptonic origin exist. In the leptonic scenarios it is commonly believed that the second component arises from electrons that suffer Inverse Compton (IC) scattering with low energy photons. The origin of the low-energy photons responsible for the IC is still unclear. The so-called Synchrotron-Self-Compton (SSC) and the External Compton (EC) are considered as possible origin of photons. In the hadronic scenarios pion decays and hadronic synchrotron emission are the processes responsible for the high-energy emission if protons are accelerated to sufficiently high energies [6].

Blazars are classified as FSRQ or BL Lac (Flat Spectrum Radio Quasars) based on the strength of the emission lines where the latest are characterized by featureless emission lines. Based on the peak of the SED, 1ES $1215+303$ is classified as an intermediate (IBL) or high frequency peaked BL Lac (HBL) [7] [8].

In this contribution, we present the long-term lightcurve derived using almost nine years of data accumulated by the Fermi-LAT satellite. This study resulted also in the detection of new flaring activities occurring on the time interval from 2015 and 2017.

This proceeding is organized as follows: Section 2 describes the Fermi-LAT detector and observations. Section 3 and 4 describe data analysis techniques and analysis results respectively. Section 5 contains a short summary and the conclusions. In this work all the errors quoted are purely statistical.

\section{Fermi-LAT}

The Fermi Gamma Ray Space Telescope mission was launched on 2008 June 11, with two instruments on board; the Large Area Telescope (LAT) and the GLAST Burst Monitor (GBM). The Fermi-LAT satellite is studying the high-energy (HE; E $>\mathscr{O}(\mathrm{MeV})$ ) sky by converting $\gamma$ rays into electron/positron pairs in one of the thin tungsten foils of the detector [9]. The LAT instrument covers the energy range from $\sim 20 \mathrm{MeV}$ up to few hundreds of $\mathrm{GeV}$ and celebrated its $9^{\text {th }}$ successful operational year in June 2017.

The data accumulated by Fermi-LAT is publicly available, released in the form of event (PH) and spacecraft (SC) files containing the lifetime information and spacecraft pointing positions. 
Since the launch of Fermi-LAT, the data is continuously updated by the LAT team using more sophisticated reconstruction techniques and Instrument Response Functions (IRFs). The latest reconstruction technique (Pass 8) leads to a substantial increase of $\gamma$-ray acceptance, i.e. high photon detection probability. This allows a better reconstruction up to $500 \mathrm{GeV}$ compared to the previous one (Pass 7).

\section{Data Analysis}

For this study we used almost nine years of data taken on survey mode on 1ES 1215+303 since the start of Fermi-LAT. The data analysis is conducted with the likelihood framework provided in the Fermi ScienceTools software package, version v10r0p5. We selected events with energies $100 \mathrm{MeV}<\mathrm{E}<500 \mathrm{GeV}$ from a $15^{\circ}$ region of interest (RoI) centered on the position of $1 \mathrm{ES}$ $1215+303$ (R.A. $=12^{\mathrm{h}} 17^{\mathrm{m}} 52^{\mathrm{s}}$, decl. $=+30^{\circ} 07^{\prime} 00^{\prime \prime}, \mathrm{J} 2000$ ).

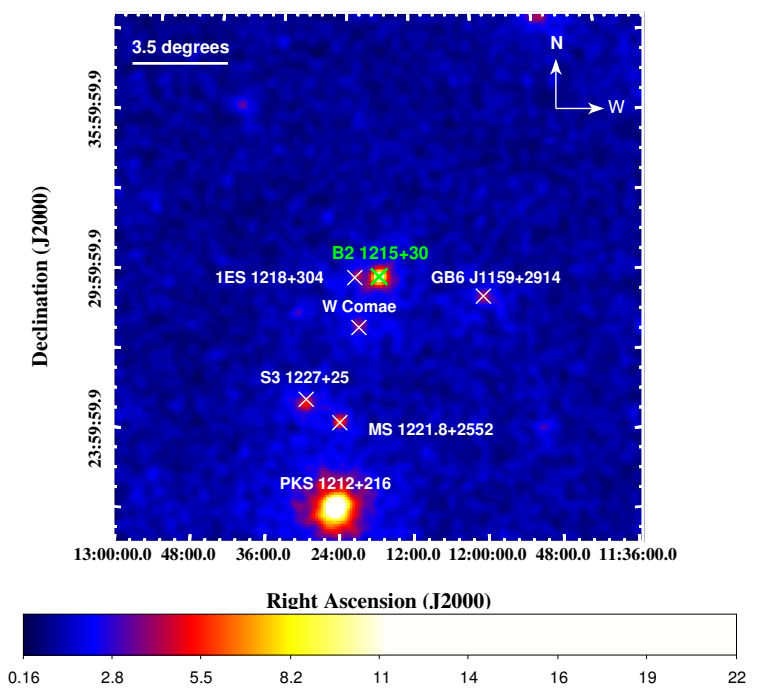

Figure 1: Counts map produced with Fermi-LAT data in a $20^{\circ} \times 20^{\circ}$ region of sky. The map is centerd at the position of 1ES $1215+303$ (marked as B2 1215+30) which is indicated a cyan cross in the map. All the other bright sources in this region are marked with a white cross.

In this analysis, the background model includes the all known $\gamma$-ray sources from the third Fermi-LAT catalog (3FGL) within the RoI and the sources located up to $5^{\circ}$ outside the RoI edges. The galactic and isotropic diffuse emissions were modeled with gll_iem_v0 6 and iso_P8R2_SOURCE_V6_V06 respectively from the Fermi ScienceTools and a set of instrument response functions P8R2_SOURCE_V6 ${ }^{1}$ were used. The contamination from $\gamma$-ray Earth's limb is taken into account by excluding events with zenith angles $<90^{\circ}$.

\section{Analysis Results}

Figure 1 shows the counts map produced using 6 months of data from 2016. It shows an RoI of $20^{\circ}$ centered on 1 ES $1215+303$ (marked as B2 1215+30). This sky region is interesting

\footnotetext{
${ }^{1}$ http://fermi.gsfc.nasa.gov/ssc/data/analysis/user/
} 
since it is a sky region populated with $\mathrm{TeV}$ sources. Only the bright sources appearing in this region are marked with a cross. All of them are BL Lac type objects and TeV emitters, except the GB6 J1159+2914 which is a quasar and no TeV emission has been detected from it so far. One can distinguish the nearby source, $1 \mathrm{ES} 1218+304$ ( $\mathrm{z}=0.182$ ), a bright $\mathrm{TeV}$ emitter located (R.A. $=12^{\mathrm{h}} 21^{\mathrm{m}} 26.3^{\mathrm{s}}$, decl. $=+30^{\circ} 11^{\prime} 29^{\prime \prime}, \mathrm{J} 2000$ ). These two nearby sources exhibit opposite emission behaviour, in terms of brightness, in the $\mathrm{GeV}$ and $\mathrm{TeV}$ energy bands. For instance, 1ES 1215+303 apperas brighter in the GeV energy range and faint in TeV, whereas 1ES 1218+304 exhibits the opposite behaviour in this regard [10].

\subsection{Long-term Lightcurve}

The lightcurve was derived using the gtlike algorithm from ScienceTools to calculate the integral flux in different time bins. The spectrum of 1ES 1215+303 was modeled using a simple power law:

$$
\frac{\mathrm{dN}}{\mathrm{dE}}=\mathrm{N}_{0}\left(\frac{\mathrm{E}}{\mathrm{E}_{0}}\right)^{-\Gamma}
$$

where $\mathrm{N}_{0}$ is the normalization factor at a chosen reference energy $\mathrm{E}_{0}$, and $\Gamma$ is the photon index. The spectral index and integral flux of 1ES $1215+303$ were let free during the global fit. Other known sources located within RoI but detected with TS $>9(\sigma \simeq \sqrt{T S})$, were kept in the model with fixed parameters to their catalog values. While calculating the integral flux in short time bins, only the integral flux was left free while the spectral index was fixed to the value obtained during the fitting procedure for the entire time range. For the other sources inside the RoI, detected with a TS $<$, all the parameters were frozen to the values obtained during the fitting procedure over the whole time period.

The long term-light curve, derived with data from September 2008 1st to 2017 June 30, corresponding to MJD (54710-57934) is shown in Figure 4. The flux's evolution of 1ES 1215+303 is studied by calculating the integral flux, from $100 \mathrm{MeV}-500 \mathrm{GeV}$ in one week time bins. Three major flaring episodes, with weekly averaged flux I $>3.0 \times 10^{-07} \mathrm{~cm}^{-2} \mathrm{~s}^{-1}$, are detected during October 2008, February 2014 and April 2017.

\begin{tabular}{cccc} 
Fermi-LAT $(0.1-500 \mathrm{GeV})$ & Dates & Signal & Flux $\left[\mathrm{cm}^{-2} \mathrm{~s}^{-1}\right]$ \\
\hline & 2008 Oct 12 - 2008 Oct 13 & $15.5 \sigma$ & $(9.2 \pm 0.15) \times 10^{-7}$ \\
& 2014 Feb 08 - 2014 Feb 09 & $12.2 \sigma$ & $(9.2 \pm 0.18) \times 10^{-7}$ \\
& 2017 Apr 12 - 2017 Apr 13 & $14.0 \sigma$ & $(8.0 \pm 0.15) \times 10^{-7}$
\end{tabular}

Table 1: The major flares of 1ES 1215+303 detected using nine years of Fermi-LAT observations

The strength of the signal during these flares allows to derive the lightcurve in one day time bins. The corresponding lightcurves in one day time bin around these flares are shown in the bottom part of Figure 4. These flares are investigated by deriving one day time bin lightcurves for a longer time period, centered around the highest flux value. The corresponding details are summarized in Table 4.1. The strength of these flares allows to investigate the variability on shorttime scales and it is possible to go down to 4.6 hours time bins.

Fermi-LAT reported on the detection of a bright flare on 2014 Feb 14, seen simultaneously by VERITAS experiment at $\mathrm{TeV}$ energies. We investigated the latest flaring activities, occurring in the $\mathrm{GeV}$ range after this date and found the source in several high flux states. 


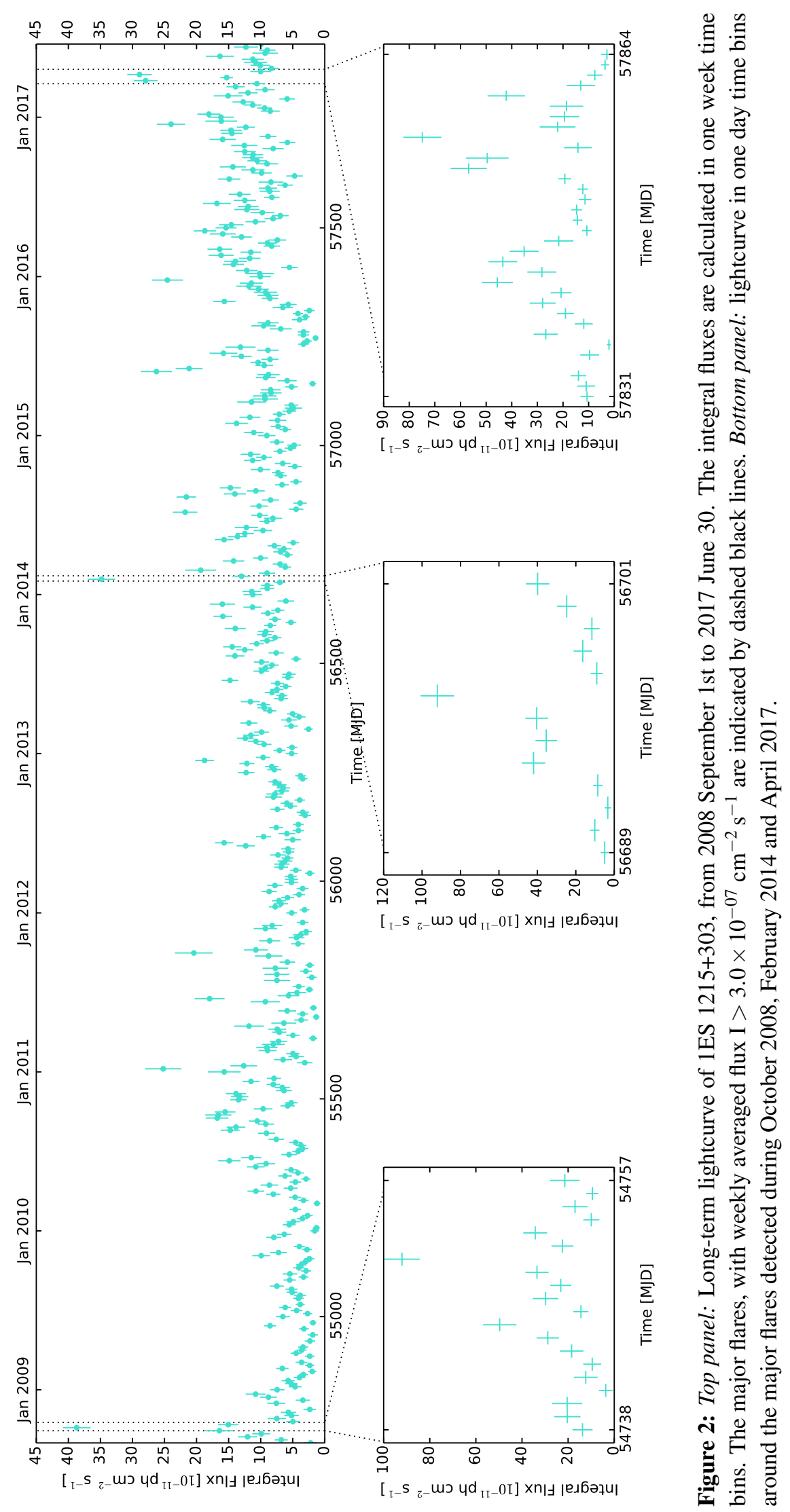




\subsection{Recent flaring activities}

This analysis resulted in the detection of several flux increament from 2015 to 2017, where only one flare has been reported by the Fermi-LAT on 2017 April 13 (ATel \#10270). The corresponding lightcurves for these years are plotted in Figure 3. The flaring episodes where the flux is exceeding the value of $\mathrm{I}>2.4 \times 10^{-8} \mathrm{~cm}^{-2} \mathrm{~s}^{-1}$ (weekly average) are summarized in Table 4.2.
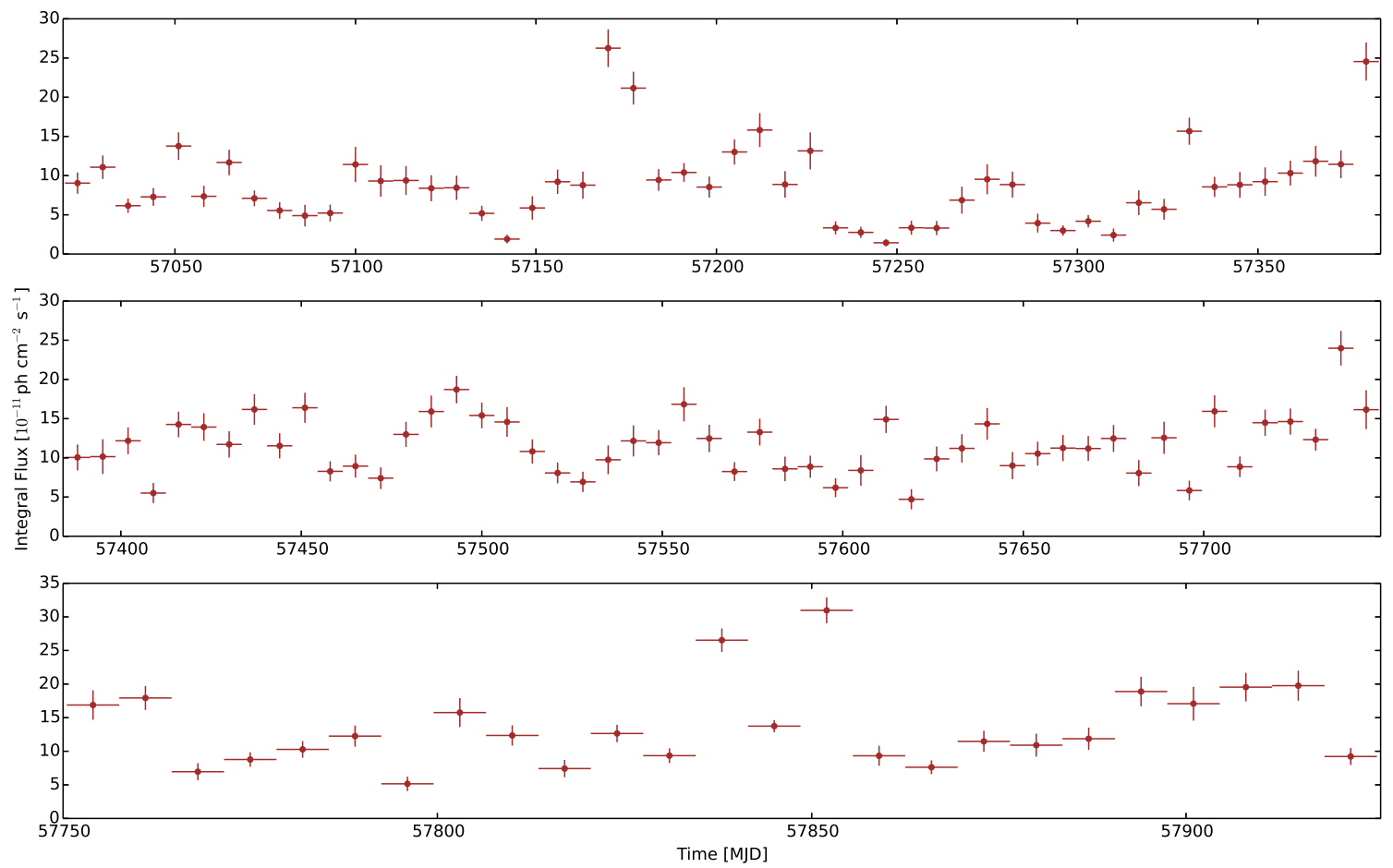

Figure 3: Integral flux I $(100 \mathrm{MeV}<\mathrm{E}<500 \mathrm{GeV})$ in one week time bins for all the 2015 (MJD 5702357387) and 2016 (MJD 57388-57753) sets in top and middle panels. The bottom panel shows six months of data, from 2017 January 1st to 2017 June 30 (MJD 57754-57934).

\begin{tabular}{cccl} 
Fermi-LAT $(0.1-500 \mathrm{GeV})$ & Dates & Signal & Flux $\left[\mathrm{cm}^{-2} \mathrm{~s}^{-1}\right]$ \\
\hline & 2015 Jan 01-2015 Jan08 & $12.0 \sigma$ & $(2.6 \pm 0.48) \times 10^{-7}$ \\
& 2015 Dec 23-2015 Dec 30 & $9.4 \sigma$ & $(2.5 \pm 0.48) \times 10^{-7}$ \\
& 2016 Dec 16-2014 Dec 23 & $14.2 \sigma$ & $(2.4 \pm 0.44) \times 10^{-7}$ \\
2017 Mar 25 - 2017 Apr 01 & $21.6 \sigma$ & $(2.7 \pm 0.35) \times 10^{-7}$ \\
2017 Apr 07 - 2017 Apr 15 & 20.1 $\sigma$ & $(3.1 \pm 0.38) \times 10^{-7}$
\end{tabular}

Table 2: The latest flares of 1ES 1215+303 detected from 2015-2017

\section{Summary and Conclusions}

In this proceeding we have presented the long-term lightcurve of the BL Lac object 1ES $1215+303$ in the energy range $100 \mathrm{MeV}<\mathrm{E}<500 \mathrm{GeV}$ with Fermi-LAT. Several flux increment have been detected from 9 years of Fermi-LAT data analysis. The major flares are reported on in this work. The three brightest flares ( $\mathrm{I}>8.0 \times 10^{-7} \mathrm{~cm}^{-2} \mathrm{~s}^{-1}$ ) occurred during 2008, 2014 and 
2017 respectively. Such events are used to derive the variability time scale and to set limits on the size of the emission region of the source.

\section{References}

[1] S. Paiano, M. Landoni, R. Falomo, A. Treves, R. Scarpa and C. Righi, On the Redshift of TeV BL Lac Objects, ApJ 837 (Mar., 2017) 144, [1701.04305].

[2] A. A. Abdo, M. Ackermann, M. Ajello, W. B. Atwood, M. Axelsson, L. Baldini et al., VizieR Online Data Catalog: Fermi/LAT bright gamma-ray source list (OFGL) (Abdo+, 2009), VizieR Online Data Catalog 218 (Nov., 2009) .

[3] J. Aleksic et al., Discovery of VHE $\gamma$-rays from the blazar 1ES 1215+303 with the MAGIC telescopes and simultaneous multi-wavelength observations, A\&A (2012), [1203.0490].

[4] A. U. Abeysekara, S. Archambault, A. Archer, W. Benbow, R. Bird, M. Buchovecky et al., A Luminous and Isolated Gamma-Ray Flare from the Blazar B2 1215+30, ApJ 836 (Feb., 2017) 205, [1701.01067].

[5] C. M. Urry and P. Padovani, Unified Schemes for Radio-Loud Active Galactic Nuclei, PASP (1995), [astro-ph/9506063].

[6] M. Boettcher, Modeling the Spectral Energy Distributions and Variability of Blazars, ArXiv e-prints (May, 2012), [1205.0539].

[7] E. Nieppola, M. Tornikoski and E. Valtaoja, Spectral energy distributions of a large sample of BL Lacertae objects, A\&A 445 (Jan., 2006) 441-450, [a stro-ph/ 050904 5].

[8] M. Ackermann, M. Ajello, A. Allafort, E. Antolini, W. B. Atwood, M. Axelsson et al., The Second Catalog of Active Galactic Nuclei Detected by the Fermi Large Area Telescope, ApJ 743 (Dec., 2011) 171, [1108.1420].

[9] W. B. Atwood, A. A. Abdo, M. Ackermann, W. Althouse, B. Anderson, M. Axelsson et al., The Large Area Telescope on the Fermi Gamma-Ray Space Telescope Mission, ApJ 697 (June, 2009) 1071-1102, [0902.1089].

[10] E. Aliu, S. Archambault, T. Arlen, T. Aune, M. Beilicke, W. Benbow et al., Long Term Observations of B2 1215+30 with VERITAS, ApJ 779 (Dec., 2013) 92, [1310 . 64 98]. 